\title{
Difficulties in Evaluating Lyapunov Exponents for Lie Governed Dynamics
}

\author{
C. M. Sarris, ${ }^{1}$ A. Plastino, ${ }^{2,3}$ and A. N. Proto ${ }^{4}$ \\ ${ }^{1}$ Ciclo Basico Comun, Catedra de Fisica, Universidad de Buenos Aires, Argentina and Laboratorio de Sistemas Complejos, \\ FI-UBA, Argentina \\ ${ }^{2}$ Instituto de Física La Plata-CCT-CONICET, Universidad Nacional de La Plata, C.C. 727, 1900 La Plata, Argentina \\ ${ }^{3}$ Physics Department and IFISC-CSIC, University of the Balearic Islands, 07122 Palma de Mallorca, Spain \\ ${ }^{4}$ Laboratorio de Sistemas Complejos, FI-UBA, Argentina and GIDyCSI-Universidad Nacional de Lanús, Argentina
}

Correspondence should be addressed to A. Plastino; angeloplastino@gmail.com

Received 26 February 2013; Revised 17 June 2013; Accepted 18 June 2013

Academic Editor: Svetoslav Nikolov

Copyright (C) 2013 C. M. Sarris et al. This is an open access article distributed under the Creative Commons Attribution License, which permits unrestricted use, distribution, and reproduction in any medium, provided the original work is properly cited.

We consider here an environment in which the fact that a semiquantum Hamiltonian obeys SU(2) symmetries poses serious difficulties if one wants to compute Lyapunov exponents.

\section{Introduction}

An extreme complexity of phase space's trajectories that are very sensitive to small changes in the initial conditions is the signature of classical chaos, accompanied by (i) an ostensibly random allotment of phase points on a Poincare's surface of section and (ii) an exponentially rapid separation of two initially close trajectories [1]. Instead, it is clear that the state vector of a closed quantum system cannot exhibit chaotic motion in Hilbert space. The interaction between a quantum system and a classical one may instead lead to authentic chaotic motion of the quantum component, a phenomenon known as semiquantum chaos [2,3]. Remark that the vocable semiquantum is reserved to systems for which neither the quantum part nor the classical part would be chaotic by themselves. If chaos ensues, this happens because of the classical-quantum coupling. Semiquantumness implies that one part is treated classically and the other one in quantal fashion [3].

We consider here an environment in which the semiquantum Hamiltonian obeys SU(2) symmetries and consider the difficulties that arise if one wants to compute Lyapunov exponents [4].

The paper is organized as follows: Section 2 deals with some background materials [5], while Section 3 explicates Hamiltonian details.

\section{Background}

Consider a system that possesses both quantum and classic degrees of freedom, with a coupling amongst them, that we call semiquantum $[3,6-10]$. The associated Hamiltonian is of the general form [11]

$$
\widehat{H}=\widehat{H}_{q}+H_{\mathrm{cl}}+H_{\mathrm{int}},
$$

where $\widehat{H}_{q}, H_{\mathrm{cl}}$, and $H_{\text {int }}$ are the quantum, classical, and interaction parts, respectively. There exist many situations in which a semiquantum description has been attempted $[6,9,12]$. Porter [6] made an exhaustive compilation of physical systems for which this kind of description is relevant. One may highlight vibrating quantum billiards as a useful abstraction of the ensuing semiquantum dynamics [13]. Indeed, many semiquantum Hamiltonians are found in the literature [3, 14-23].

In this work, via the Maximum Entropy Principle (MEP) vantage point [5], we show that serious difficulties arise, in the case of these systems, if one wants to compute Lyapunov exponents, because the quantum degrees of freedom of the system must abide by the generalized uncertainty principle (GUP) [24] which in the case of an underlying SU(2) Lie algebra becomes a dynamic invariant. In turn, the other dynamic invariants (the energy) involve both quantum and 
classical degrees of freedom. Accordingly, the initial condition pertaining to at least one of the classical degrees of freedom will depend not only upon the energy value but also on the uncertainty relation as well. This will be illustrated below.

These invariants allow us to (i) adequately define initial conditions and (ii) follow the details of the temporal evolution, from regular regimes to irregular ones. Our main invariant is none other than the generalized uncertainty principle (GUP) [24]. From a MEP viewpoint, in the analysis of a semiquantum dynamics, one deals with a peculiar dynamic "working" space spanned by the variables $\left\langle\widehat{O}_{1}\right\rangle, \ldots,\left\langle\widehat{O}_{N}\right\rangle$, $q_{1}, \ldots, q_{n}, p_{1}, \ldots, p_{n}$. The first $N$ variables $\left\langle\widehat{O}_{1}\right\rangle, \ldots,\left\langle\widehat{O}_{N}\right\rangle$ are the mean values of a set of noncommuting observable closing a partial Lie semialgebra under commutation with the Hamiltonian. We insist on the fact that these quantum variables must obey the uncertainty relation not only at the instant $t=0$ but also for any other $t$. The $q_{1}, \ldots, q_{n}$, $p_{1}, \ldots, p_{n}$ are $2 n$ classical variables of the system. As in the full quantum case $[25,26]$, we will see for semiquantum $S U(2)$ nonlinear dynamics; the closure condition defines an $N \times N$ dynamic matrix $G\left(q_{i}, p_{i}\right)$ which governs the dynamics of the system's quantum degrees of freedom. This matrix is now of a semiquantal nature since it also depends upon classical degrees of freedom.

We follow the prescription given in $[8,9,11,12,18,19]$; that is, the energy of the system is taken to coincide with the expectation value of the Hamiltonian, $\langle\widehat{H}\rangle=\operatorname{Tr}(\widehat{\rho} \widehat{H})$, traced over the quantum state, $\widehat{\rho}$. In turn, $\langle\widehat{H}\rangle$ generates the time evolution of the classical degrees of freedom in the orthodox classical mechanics' fashion. The MEP's point of view approach to semiquantal systems takes advantage of two facts:

(1) it is possible to describe the time evolution of the quantum degrees of freedom in the dual space of Lagrange multipliers associated to the quantum observable [25-27] and

(2) the existence of the GUP invariant, $I^{H}$ say, makes it possible to analyze the dynamics of the system in different regimes (irregular and regular) by varying $I^{H}$ 's value through the initial conditions of the system.

\section{Quantum Plus Classical Hamiltonians and Maximum Entropy Approach}

Consider the Hamiltonian (1). The classical degrees of freedom are the canonical conjugate variables $\left(q_{i}, p_{i}\right)$. We choose for the Hamiltonian (1) the specific form

$$
\widehat{H}=\sum_{j} \sum_{i=1}^{n} a_{j}\left(q_{i}, p_{i}\right) \widehat{O}_{j}+\sum_{i=1}^{n} F\left(q_{i}, p_{i}\right) \text {, }
$$

where the first term includes both the $\widehat{H}_{q}$ (nonchaotic) and $H_{\text {int }}$ (chaos-generator) ingredients, with the $\widehat{O}_{j}$ 's being quantum operators, while the last term is a purely classical and nonchaotic, with $F\left(q_{i}, p_{i}\right)$ functions of the canonically conjugate classical variables $\left(q_{i}, p_{i}\right)$. We have in fact a family of Hamiltonians with a classical phase-space substratum.

The MEP formalism [25-27] deals with the quantum degrees of freedom of the systems (2) and provides a density operator $\rho(t)$ [25-27]

$$
\widehat{\rho}(t)=\exp \left(-\lambda_{0} \widehat{I}-\sum_{j=1}^{N} \lambda_{j}(t) \widehat{O}_{j}\right),
$$

in terms of $N+1$ Lagrange multipliers $\left(\lambda\right.$ 's), where $\widehat{O}_{0}=\widehat{I}$ is the identity operator and $\lambda_{0}=\operatorname{Tr}\left[\exp \left(-\sum_{j=1}^{N} \lambda_{j}(t) \widehat{O}_{j}\right)\right]$ is determined through the normalization condition. Alhassid and Levine demonstrated $[25,26]$ that the sufficient number $N$ of constraints

$$
\langle\widehat{O}\rangle=\operatorname{Tr}[\widehat{\rho}(t) \widehat{O}]
$$

Is necessary in order for the state operator (3) to be valid for any time $t$ deriveing from the closure condition

$$
\left[\widehat{H}(t), \widehat{O}_{j}\right]=i \hbar \sum_{r=0}^{N} g_{r j}(t) \widehat{O}_{r}, \quad j=1,2, \ldots, N .
$$

Equation (5) defines a $N \times N$ matrix $G(t)$ whose coefficients $g_{r j}(t)$ may depend upon the time if the Hamiltonian $\widehat{H}(t)$ of the system is time dependent. With the help of (5) and considering that the set of relevant operators $\left\{\widehat{O}_{1} ; \ldots ; \widehat{O}_{N}\right\}$ defined through (5) are linearly independent, it is possible to obtain the equations of motion of the Lagrange multipliers [25-27] (see (8)) below. Note that (5) involves the classical variables $\left(q_{i}, p_{i}\right)$ in the guise of parameters, on account of the coefficients $a_{j}\left(q_{i}, p_{i}\right)$ intervening in (2). Thus, (5) adopts the appearance

$$
\left[\widehat{H}, \widehat{O}_{k}\right]=i \hbar \sum_{r=0}^{N} \sum_{i=1}^{n} g_{r j}\left(q_{i}, p_{i}\right) \widehat{O}_{r}
$$

with $k=1, \ldots, N$. The $\widehat{O}_{k}$ 's are $N$ quantum operators closing a partial Lie algebra under commutation with $(2)[25,26]$. The $g_{r j}\left(q_{i}, p_{i}\right)$ defines a semiquantum matrix $G\left(q_{i}, p_{i}\right)$.

Since we are going to describe the quantum state of the system (2) by means of the quantum statistical operator given by (3), it is required that this operator be represented by (3) for all $t$ so that the entropy $S[25,26]$

$$
S=\lambda_{0}+\sum_{j=1}^{N} \lambda_{j}(t)\left\langle\widehat{O}_{j}\right\rangle(t),
$$

is a constant of the motion $[25,26]$. The Lagrange multipliers, in turn, fulfill the following equations of motion (see [22] for more details):

$$
\frac{d \lambda_{k}}{d t}=\sum_{r=1}^{N} \sum_{i=1}^{n} g_{k r}\left(q_{i}, p_{i}\right) \lambda_{k}(t), \quad k=1, \ldots, N
$$

The semiquantum matrix $G\left(q_{i}, p_{i}\right)$ defined through (6) enables us to obtain the equations of motion of the mean values for the $N$ quantum degrees of freedom via [22]

$$
\frac{d\left\langle\widehat{O}_{k}\right\rangle}{d t}=-\sum_{r=1}^{N} \sum_{i=1}^{n} g_{r k}\left(q_{i}, p_{i}\right)\left\langle\widehat{O}_{r}\right\rangle, \quad k=1, \ldots, N \text {. }
$$


Equations (8) and (9) display the interplay between the quantum and classical degrees of freedom. In order to describe our classical degrees of freedom, we need to know the equation of motion for the $2 n$ classical variables $\left(q_{i}, p_{i}\right)$.

To do so, we consider the mean value of (2), traced over the quantum state (3), as a classical Hamiltonian function so that $\langle\widehat{H}\rangle=\operatorname{Tr}(\widehat{\rho} \widehat{H})$ generates the temporal evolution of the classical degrees of freedom $[8,9,11,12,18,19]$

$$
\begin{array}{ll}
\frac{d q_{i}}{d t}=\left\{q_{i},\langle\widehat{H}\rangle\right\}, & i=1, \ldots, n, \\
\frac{d p_{i}}{d t}=\left\{p_{i},\langle\widehat{H}\rangle\right\}, & i=1, \ldots, n,
\end{array}
$$

where $\{\cdot\}$ indicates Poisson brackets.

The generalized uncertainty principle (GUP) in the present framework reads [24]

$$
\begin{aligned}
& I^{H}= \sum_{j=1}^{N} \sum_{\substack{k=1 \\
j<k}}^{N}\left\{\left(\Delta \widehat{O}_{j}\right)^{2}\left(\Delta \widehat{O}_{k}\right)^{2}\right. \\
&\left.-\left[\frac{1}{2}\left\langle\widehat{O}_{j} \widehat{O}_{k}+\widehat{O}_{k} \widehat{O}_{j}\right\rangle-\left\langle\widehat{O}_{j}\right\rangle\left\langle\widehat{O}_{k}\right\rangle\right]^{2}\right\} \\
& \geq-\frac{1}{4} \sum_{j=1}^{N} \sum_{\substack{k=1 \\
j<k}}^{N}\left\langle\left[\widehat{O}_{i}, \widehat{O}_{j}\right]\right\rangle .
\end{aligned}
$$

The GUP relationship given by (11) is obtained as a sum over the principal minors (of order 2) of the correlation matrix $K(t)$, corresponding to the observable set defined by the closure condition (6). The correlation matrix is a definite positive one, and its elements are defined via [28]

$$
K_{i j}(t)=\frac{1}{2}\left\langle\widehat{O}_{i} \widehat{O}_{j}+\widehat{O}_{j} \widehat{O}_{i}\right\rangle_{(t)}-\left\langle\widehat{O}_{i}\right\rangle_{(t)}\left\langle\widehat{O}_{j}\right\rangle_{(t)} .
$$

3.1. The $S U(2)$ Instance. It is well known that $\left\{\widehat{\sigma}_{x}, \widehat{\sigma}_{y}, \widehat{\sigma}_{z}\right\}$ is a basis of the SU(2) algebra and the following commutation relationship [29] holds:

$$
\left[\widehat{\sigma}_{j}, \widehat{\sigma}_{k}\right]=2 i \varepsilon_{j k l} \widehat{\sigma}^{l}=2 i \sum_{l=1}^{3} \varepsilon_{j k l} \widehat{\sigma}_{l},
$$

and the semiquantum Hamiltonian of the type (2) becomes

$$
\widehat{H}=\sum_{j=1}^{3} \sum_{i=1}^{n} a_{j}(q, p) \widehat{\sigma}_{j}+\frac{p^{2}}{2 m}+V(q),
$$

where $\widehat{\sigma}_{j}$ are the generators of $\mathrm{SU}(2)$.

Proposition 1. If a set of operators, which fulfills the commutation relation (13), closes a commutation algebra with a Hamiltonian of the type (14), then the semiquantum matrix $G\left(q_{i}, p_{i}\right)$ of the system, defined by means of the closure condition (6), is an antisymmetric one.
Every Hamiltonian that closes an algebra with the SU(2) generators is accompanied by the GUP invariant (11) which for the SU(2) Lie algebra adopts the fashion [24] consider the following:

$$
I^{H}=3-2\left[\left\langle\widehat{\sigma}_{x}\right\rangle^{2}+\left\langle\widehat{\sigma}_{y}\right\rangle^{2}+\left\langle\widehat{\sigma}_{z}\right\rangle^{2}\right]=3-2\langle\widehat{\sigma}\rangle^{2} .
$$

Because of the uncertainty principle (11), $I^{H} \geq$ $(-1 / 4) \sum_{\substack{j, k=1 \\ j<k}}^{q}\left\langle\left[\widehat{\sigma}_{j}, \widehat{\sigma}_{k}\right]\right\rangle^{2}$, and Schwarz' inequality entails $\langle\widehat{\sigma}\rangle^{2} \leq 1$, that is, the uncertainty principle for the SU(2) Lie algebra, that can be expressed in the guise

$$
0<\left\langle\widehat{\sigma}_{x}\right\rangle^{2}+\left\langle\widehat{\sigma}_{y}\right\rangle^{2}+\left\langle\widehat{\sigma}_{z}\right\rangle^{2}<1,
$$

defining the celebrated Bloch sphere of the system.

\section{First Difficulty: The Normalization Difficulty}

In evaluating Lyapunov exponents (LE), we must consider the action of the motion invariants (MI). Any "orbit" calculation must "respect them." In particular, we must take care of not violating the uncertainty principle as the system evolves (same for the normalization procedure) in the process of Lyapunov exponents' evaluation. Once we have chosen a GUP-value at $t=0$ via (16), this value must remain the same at any other time $t$ so as not to violate the uncertainty principle. More specifically, we are speaking of the fiduciary trajectory and of the ones infinitely close to it that one keeps renormalizing when the Euclidean distance between them grows. If the uncertainty principle ((11) or (16)) is violated during the normalization procedure, the concept of semiquantum system (2) will become a mere "mathematical artifact" (in the words of [3]) but cease to have physical meaning. To illustrate what we mean, we consider the semiquantum SU(2) Hamiltonian [3]

$$
\widehat{H}=B \widehat{\sigma}_{z}+C q \widehat{\sigma}_{x}+\frac{p^{2}}{2 m}+\frac{q^{4}}{4},
$$

whose equations of motion for quantum degrees of freedom (mean values and Lagrange multipliers) and classical degrees of freedom are

$$
\begin{gathered}
\frac{d\left\langle\widehat{\sigma}_{x}\right\rangle}{d t}=-2 B\left\langle\widehat{\sigma}_{y}\right\rangle, \\
\frac{d\left\langle\widehat{\sigma}_{y}\right\rangle}{d t}=2 B\left\langle\widehat{\sigma}_{x}\right\rangle-2 C q\left\langle\widehat{\sigma}_{z}\right\rangle, \\
\frac{d\left\langle\widehat{\sigma}_{z}\right\rangle}{d t}=2 C q\left\langle\widehat{\sigma}_{y}\right\rangle \\
\frac{d \lambda_{x}}{d t}=-2 B \lambda_{y}, \\
\frac{d \lambda_{y}}{d t}=2 B \lambda_{x}-2 C q \lambda_{z}
\end{gathered}
$$




$$
\begin{gathered}
\frac{d \lambda_{z}}{d t}=2 C q \lambda_{y} . \\
\frac{d q}{d t}=\frac{p}{m}, \\
\frac{d p}{d t}=-C\left\langle\widehat{\sigma}_{x}\right\rangle-q^{3} .
\end{gathered}
$$

Our motion invariants (MIs) for the system given by (17) are [5]

(i) generalized uncertainty principle (GUP) (see (11))

$$
\operatorname{GUP}(t=0)=\langle\sigma\rangle_{(0)}^{2}=\left\langle\widehat{\sigma}_{x}\right\rangle_{(0)}^{2}+\left\langle\widehat{\sigma}_{y}\right\rangle_{(0)}^{2}+\left\langle\widehat{\sigma}_{z}\right\rangle_{(0)}^{2},
$$

(ii) energy $(\operatorname{Tr}(\widehat{\rho} \widehat{H}))$

$$
E(t=0)=B\left\langle\widehat{\sigma}_{z}\right\rangle_{(0)}+C q_{(0)}\left\langle\widehat{\sigma}_{x}\right\rangle_{(0)}+\frac{p_{(0)}^{2}}{2 m}+\frac{q_{(0)}^{4}}{4},
$$

(iii) evolution vector

$$
u(t=0)=\lambda_{x(0)}\left\langle\widehat{\sigma}_{x}\right\rangle_{(0)}+\lambda_{y(0)}\left\langle\widehat{\sigma}_{y}\right\rangle_{(0)}+\lambda_{z(0)}\left\langle\widehat{\sigma}_{z}\right\rangle_{(0)},
$$

(iv) entropy $(-\operatorname{Tr}(\hat{\rho} \ln \hat{\rho}))$

$$
S(t=0)=\lambda_{0}+\lambda_{x(0)}\left\langle\widehat{\sigma}_{x}\right\rangle_{(0)}+\lambda_{y(0)}\left\langle\widehat{\sigma}_{y}\right\rangle_{(0)}+\lambda_{z(0)}\left\langle\widehat{\sigma}_{z}\right\rangle_{(0)} \text {, }
$$

with

$$
\begin{gathered}
\lambda_{0}=\ln \left[2 \cosh \left(\sqrt{\lambda_{x}^{2}+\lambda_{y}^{2}+\lambda_{z}^{2}}\right)\right], \\
\Lambda=\sqrt{\lambda_{x}^{2}+\lambda_{y}^{2}+\lambda_{z}^{2}},
\end{gathered}
$$

invariant in $\lambda$-space whose value is not an arbitrary one. The $\lambda$ 's values are adjusted after setting initial conditions (IC) for the UP $\langle\sigma\rangle_{(0)}^{2}$, since the invariants' expressions in the two reciprocal spaces (1) expectation values one and (2) $\lambda$ one are related by [5]

$$
\langle\widehat{\sigma}\rangle^{2}=\tanh ^{2}\left(\sqrt{\lambda_{x}^{2}+\lambda_{y}^{2}+\lambda_{z}^{2}}\right) .
$$

All these invariants' values are closely linked to the uncertainty principle's value given by (26) and, therefore, cannot be chosen disregarding it. Accordingly, one must mind the evolution equations for $\left\langle\widehat{\sigma}_{x}\right\rangle,\left\langle\widehat{\sigma}_{y}\right\rangle,\left\langle\widehat{\sigma}_{z}\right\rangle, q, p, \lambda_{x}, \lambda_{y}$, and $\lambda_{z}$ given by (18)-(25). Let $X=\left(\left\langle\widehat{\sigma}_{x}\right\rangle,\left\langle\widehat{\sigma}_{y}\right\rangle,\left\langle\widehat{\sigma}_{z}\right\rangle, q, p\right)$ be a vector field belonging to the solution space of (18)-(20) and (24)(25) and let $\left\{T^{t}\right\}$ be the flow induced by $X$ [4], that is, as it reads in [4]: "for any $t$ let $T^{t} x=x(t)$, where $\{x(t)\}$ is an integral curve of the vector field $X$ such that $x(0)=x$." Now let us choose two neighboring initial conditions $x$ and $y$ not lying on the same trajectory as prescribed by [4], but minding the conservation of the invariants given by (26) to (31) over the whole evolution process. Thus, we choose

$$
\begin{gathered}
x=x(0)=\left(\left\langle\widehat{\sigma}_{x}\right\rangle_{(0)},\left\langle\widehat{\sigma}_{y}\right\rangle_{(0)},\left\langle\widehat{\sigma}_{z}\right\rangle_{(0)}, q_{(0)}, p_{(0)}\right), \\
y=\left(\left\langle\widehat{\sigma}_{x}\right\rangle^{\prime},\left\langle\widehat{\sigma}_{y}\right\rangle^{\prime},\left\langle\widehat{\sigma}_{z}\right\rangle^{\prime}, q^{\prime}, p^{\prime}\right) .
\end{gathered}
$$

In (33) and (34), the initial conditions must be set as follows: (i) choose any value for our GUP invariant (26) that it does not violate the uncertainty principle (16), that is, $0<\left\langle\widehat{\sigma}_{x}\right\rangle_{(0)}^{2}+$ $\left\langle\widehat{\sigma}_{y}\right\rangle_{(0)}^{2}+\left\langle\widehat{\sigma}_{z}\right\rangle_{(0)}^{2}<1$. Once this value is chosen, it must remain the same during the whole evolution of the system not only for the fiduciary orbit (33) but also for any other infinitely close to it that one keeps renormalizing (in this way, we assure that the quantum state is always evolving on the same Bloch sphere, i.e., the same radius, in order not to violate the uncertainty principle, see (34)); (ii) choose arbitrarily two of the three following values: $\left\langle\widehat{\sigma}_{x}\right\rangle_{(0)},\left\langle\widehat{\sigma}_{y}\right\rangle_{(0)}$, or $\left\langle\widehat{\sigma}_{z}\right\rangle_{(0)}$, and the third must be obtained from the relationship given by (26); (iii) choose a value for the energy $E_{(0)}=\langle\widehat{H}\rangle_{(0)}$ which is also another invariant of the motion; (iv) choose an initial value for one of the classical variables, for example, $q_{(0)}$; (v) it is clear that the $p_{(0)}$ value cannot be fixed at will since $p_{(0)}$ is of the form

$$
p_{(0)}=\sqrt{2 m\left(E_{(0)}-B\left\langle\widehat{\sigma}_{z}\right\rangle_{(0)}-C q_{(0)}\left\langle\widehat{\sigma}_{x}\right\rangle_{(0)}-\frac{q_{(0)}^{4}}{4}\right)}
$$

and its initial value is constrained to the $E_{(0)}$ value and to the uncertainty principle value! This is a curious feature of the semiquantum dynamics: the values of the classical degrees of freedom are influenced by the uncertainty principle followed by the quantum degrees of freedom of the system (see [22] for more details). The steps (i) to (v) given above give conditions to be followed by not only on the fiducial trajectory (33) but also by all trajectories infinitely close to it. Otherwise, the uncertainty principle will be violated. With such restrictions in mind, we choose $\left\langle\widehat{\sigma}_{x}\right\rangle^{\prime}=\left\langle\widehat{\sigma}_{x}\right\rangle_{(0)}$; $\left\langle\widehat{\sigma}_{y}\right\rangle^{\prime}=\left\langle\widehat{\sigma}_{y}\right\rangle_{(0)} ;\left\langle\widehat{\sigma}_{z}\right\rangle^{\prime}=\left\langle\widehat{\sigma}_{z}\right\rangle_{(0)} ; q^{\prime}=q_{(0)}+10^{-6} ; p^{\prime}=$ $\sqrt{2 m\left(E_{(0)}-B\left\langle\widehat{\sigma}_{z}\right\rangle^{\prime}-C q^{\prime}\left\langle\widehat{\sigma}_{x}\right\rangle^{\prime}-q^{\prime} 4 / 4\right)}$.

We denote, as in ([4]), by $d$ the length of the segment joining $x$ to $y(|d|)$ at $t_{0}=0$, so that

$$
|d|=\|x-y\|=\|x(0)-y\|,
$$

where $\|\cdots\|$ is the Euclidean norm. Let $x_{1}=T^{\tau} x(0)$ and $\left|d_{1}\right|=\left\|T^{\tau} x(0)-T^{\tau} y\right\|$ [4]. Reference [4] prescribes: "denote by $y_{1}$ the unique point on the half-line issuing from $x_{1}$ and containing $T^{\tau} y$ such that $\left\|y_{1}-x_{1}\right\|=|d|$ " in order to be able to iterate this procedure and define $x_{2}=T^{\tau} x_{1}=T^{2 \tau} x(0)$ and $\left|d_{2}\right|=\left\|T^{\tau} x_{1}-T^{\tau} y_{1}\right\|$ "and find the unique point on the halfline issuing from $x_{2}=T^{2 \tau} x(0)$ and containing $T^{\tau} y_{1}$ such that $\left\|y_{2}-x_{2}\right\|=|d|$ " in order to get a sequence $\left\{\left|d_{i}\right|\right\}_{i \geq 1}$ which enables one to evaluate $\lim _{n \rightarrow+\infty}\left((1 / n \tau) \sum_{i=1}^{n} \ln \left(\left|d_{i}\right| /|d|\right)\right)$ [4].

However, here the SU(2) nonlinear semiquantum dynamics reigns. We must ensure that those required points $y_{1}, y_{2}, \ldots, y_{n}$ are to be chosen in such a way that the invariants given by (26) to (31) are always respected. How could one ensure that imposing on the system the normalization condition $\left\|y_{i}-x_{i}\right\|=|d|$ at every step $\tau$ does not violate the uncertainty principle? Enters now our crucial caveat: this prescription may be inconsistent with that given by $\| y_{i}-$ $x_{i}\|=|d|=\| x(0)-y \|$. Such constraint does not exist 


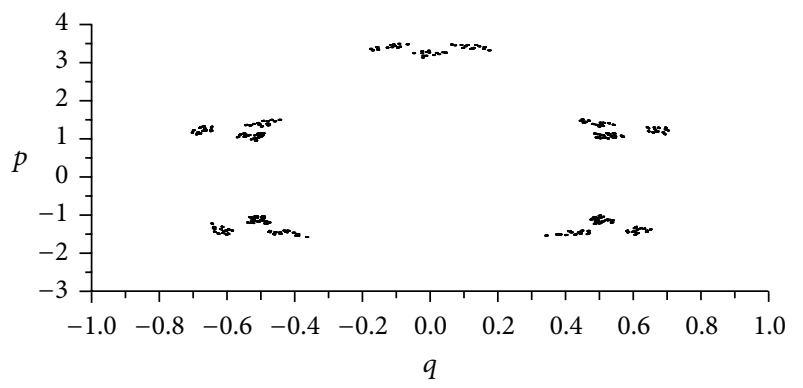

(a)

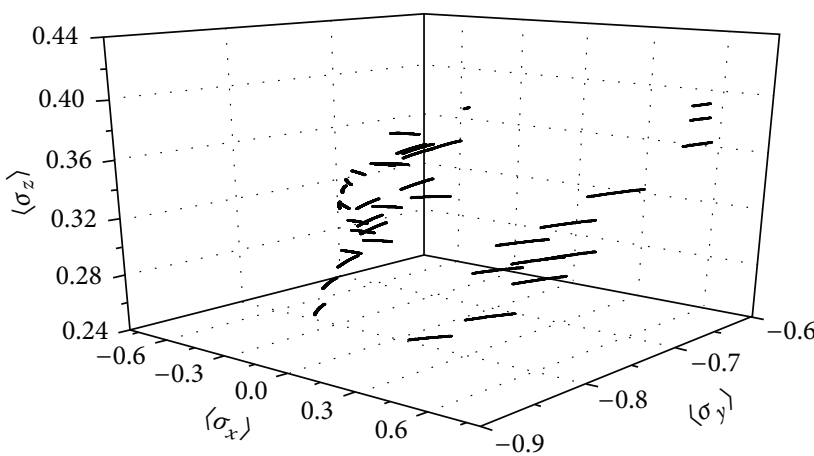

(b)

FIGURE 1: Poincaré surface of section and Bloch sphere for a SU(2) Hamiltonian and for given integration path (see text).

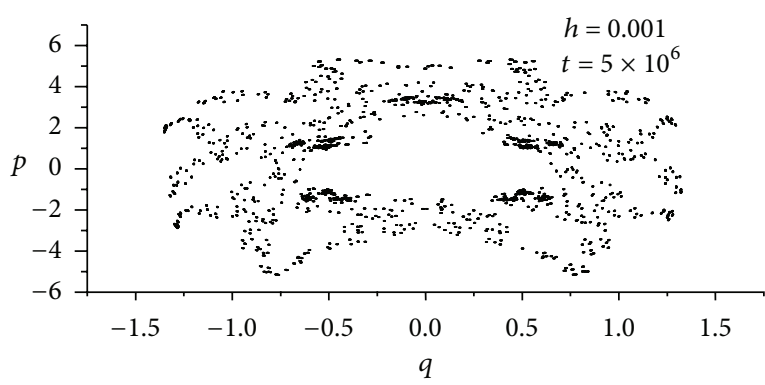

(a)



(b)

FIGURE 2: Same as Figure 1 for the same integration path but a different end point (see text).

for a classical system since there is no obligation to strictly abide by the uncertainty principle. Such an obligation is not a mere mathematical feature but a strong physical constraint $\left\|y_{i}-x_{i}\right\|=|d|=\|x(0)-y\|$. Unfortunately, all we can expect is to be able to find a point $y_{1}$ (or $y_{i}$ for each iteration) connecting $x_{1}$ to $T^{\tau} y$ with a distance from $x_{1}$ as small as possible, such that the function $\left\|y_{1}-x_{1}\right\|$ is minimal. This $y_{i}$ must comply with the uncertainty principle (a dynamic invariant for the $\mathrm{SU}(2)$ Lie algebra) whose value was set at $t_{0}=0$ by means of the initial conditions.

Summing up, in order to find the initial condition $y_{1}=\left(\left\langle\widehat{\sigma}_{x}\right\rangle_{1},\left\langle\widehat{\sigma}_{y}\right\rangle_{1},\left\langle\widehat{\sigma}_{z}\right\rangle_{1}, q_{1}, p_{1}\right)$ of the fiduciary's very close trajectory (in each iteration path), we MUST externalize the distance function $|d|=\left\|x_{1}-y_{1}\right\|$, subject to the following constraints:

(i) $y_{1}$ should respect the fixed value of GUP invariant $\operatorname{GUP}(t=0)=\langle\sigma\rangle_{(0)}^{2}($ see $(26))$ so as not to violate the uncertainty principle,

(ii) $y_{1}$ does the same with the invariant $E(0)$ (see (27)) so as not to violate energy conservation,

(iii) $y_{1}$ does the same with the invariant $S(0)$ (see (29)) so as not to violate entropy conservation,

(iv) $y_{1}$ does the same with the invariant $u(0)$ (see (28)) so as not to violate the conservation of the evolution vector, (v) $y_{1}$ must lie in the half-line starting at $x_{1}$ and contain$\operatorname{ing} T^{\tau} y$.

If we do mind all this panoply at each iteration path, we can never be sure that we are evaluating $\lim _{n \rightarrow+\infty}\left((1 / n \tau) \sum_{i=1}^{n} \ln \left(\left|d_{i}\right| /|d|\right)\right)$ in a correct fashion. In general, we face an insurmountable task.

\section{Second Difficulty: Numerical Simulations' Cumulative Error}

If we evaluate Lyapunov exponents with the so-called one step method Runge-Kutta 4 (RK4), whose local truncation error is of the order $\mathrm{O}\left(h^{5}\right)$ (given that RK4 is a fourth order $\mathrm{O}\left(h^{4}\right)$ method), we face the following situation: suppose we choose an integration path $h=0.001$. This entails that our approximation to the actual solution starts off (at $t=0$ ) with a local truncation error $\left|T_{1}\right| \sim h^{4} \sim 10^{-12}$. However, as pointed out by [30], it is necessary to take into account the error arising as a consequence of cumulative effects coming from all local truncation errors, namely, the global truncation error $\left|e_{n}\right|$. After a few millions of iteration paths, the global truncation error is so important that the temporal series' approximation that we have obtained $\left\{X_{n}(t)\right\}_{i \geq 1}$ becomes meaningless. It is the same for the swarm of points in Poincaré surfaces of section. Accordingly, such evaluation 
of the Lyapunov exponents numerical is also meaningless (remember that even if the normalized trajectory begins at every $\tau_{i}$ and does not accumulate much error, the fiduciary trajectory is evolving from $t=0$ and does accrue a large global cumulative truncation error $\left.\left|e_{n}\right|\right)$. To illustrate our claim, let us return to the $\mathrm{SU}(2)$ semiquantum Hamiltonian given by (17): if we take $B=0.5 ;\langle\widehat{H}\rangle_{(0)}=0.5 ; m=16 ; C=$ $1 ;\left\langle\widehat{\sigma}_{x}\right\rangle_{(0)}=\left\langle\widehat{\sigma}_{y}\right\rangle_{(0)}=0 ;\left\langle\widehat{\sigma}_{z}\right\rangle_{(0)}=0.9 ; q_{(0)}=0.5 ; p_{(0)}=$ $\sqrt{2 m\left(E_{(0)}-B\left\langle\widehat{\sigma}_{z}\right\rangle_{(0)}-C q_{(0)}\left\langle\widehat{\sigma}_{x}\right\rangle_{(0)}-q 4_{(0)} / 4\right)}$, an integration path $h=0.001$, and integrate (18)-(20) and (24)-(25), using RK4 from $t=0$ to $t=t=10^{6}$, we obtain the Poincare surface of section $p$ versus $q$ and the Bloch sphere of Figure 1, while if we do the same from $t=0$ to $t=5 \times 10^{6}$, using the same integration path, we obtain the Poincaré surface of section $p$ versus $q$ and Bloch sphere depicted in Figure 2.

\section{Conclusions}

We have shown that the evaluation of Lyapunov exponents on semiquantum systems like (17) may become an impossible task given that these kinds of Hamiltonian systems contain both a classical component and a quantum component which must obey the uncertainty principle. We appreciate via (18)(25) the interplay between classical and quantum degrees of freedom. This means that once (i) the GUP $(t=0)=$ $\langle\sigma\rangle_{(0)}^{2}$ value is chosen (without violating the uncertainty principle) together with (ii) the energy value $E_{(0)}$ (another dynamic invariant), the classical variables become strongly constrained in the values they can attain by two facts: (i) the constant energy of the system determines the energetically accessible regions (this fact is shared by classical Hamiltonians) and (ii) the uncertainty principle, that imposes strong restrictions on their evolution (see [22] for more details). Thus, the $p_{(0)}$ value cannot be fixed without minding the condition $0<\left\langle\widehat{\sigma}_{x}\right\rangle_{(0)}^{2}+\left\langle\widehat{\sigma}_{y}\right\rangle_{(0)}^{2}+\left\langle\widehat{\sigma}_{z}\right\rangle_{(0)}^{2}<1$ that follows from the uncertainty principle. This fact is encountered only in dealing with semiquantum dynamics. Accordingly, one does not preserve the uncertainty principle's dynamic invariant value GUP $(t=0)=\langle\sigma\rangle_{(0)}^{2}$ and one will get nonsensical results. This is a distinctive feature of semiquantum dynamics that has no classical counterpart. Evaluating Lyapunov exponents for semiquantum systems is an entirely different problem for semiquantum systems than for classical ones.

In other words, as the MEP approach is able to convert the evolution equation of motion of the quantum state $\hat{\rho}(t)$ of a semiquantum system into a set of nonlinear semiquantum differential equations (see [21, 22] for more details), the quantum nature of the system rises its head in setting the initial conditions for the quantum degrees of freedom. One must not violate the uncertainty principle. At every $\tau$ when we obtain the values $y_{i}$ infinitely close to the fiduciary trajectory, we must be sure that the system abides by the uncertainty principle from the very beginning. In this sense, calculating the Lyapunov exponents for a semiquantum system is in no way a similar task to that of doing it classically.

\section{Acknowledgments}

This work was partially supported by the Project PIP1177 of CONICET (Argentina) and the Projects FIS2008-00781/FIS (MICINN)-FEDER (EU) (Spain, EU).

\section{References}

[1] A. J. Lichtenberg and M. A. Lieberman, Regular and Chaotic Dynamics, Springer, New York, NY, USA, 1992.

[2] R. Bluemel and W. P. Reinhardt, Chaos in Atomic Physics, Cambridge University Press, Cambridge, Mass, USA, 1997.

[3] L. E. Ballentine, "Is semiquantum chaos real?" Physical Review E, vol. 63, no. 5, Article ID 056204, 7 pages, 2001.

[4] G. Benettin, L. Galgani, and J.-M. Strelcyn, "Kolmogorov entropy and numerical experiments," Physical Review A, vol. 14, no. 6, pp. 2338-2345, 1976.

[5] C. M. Sarris and A. N. Proto, "Generalized metric phase space for quantum systems and the uncertainty principle," Physica A, vol. 377, no. 1, pp. 33-42, 2007.

[6] M. A. Porter, "Nonadiabatic dynamics in semiquantal physics," Reports on Progress in Physics, vol. 64, no. 9, pp. 1165-1189, 2001.

[7] R. Blumel and B. Esser, "Quantum chaos in the BornOppenheimer approximation," Physical Review Letters, vol. 72, no. 23, pp. 3658-3661, 1994.

[8] H. Schanz and B. Esser, "Mixed quantum-classical versus full quantum dynamics: coupled quasiparticle-oscillator system," Physical Review A, vol. 55, no. 5, pp. 3375-3387, 1997.

[9] A. M. Kowalski, A. Plastino, and A. N. Proto, "Semiclassical model for quantum dissipation," Physical Review E, vol. 52, no. 1, pp. 165-177, 1995.

[10] J. Ma and R. K. Yuan, "Semiquantum Chaos," Journal of the Physical Society of Japan, vol. 66, no. 8, pp. 2302-2307, 1997.

[11] A. M. Kowalski, M. T. Martin, J. Nuñez, A. Plastino, and A. N. Proto, "Quantitative indicator for semiquantum chaos," Physical Review A, vol. 58, no. 3, pp. 2596-2599, 1998.

[12] A. M. Kowalski, A. Plastino, and A. N. Proto, "A semiclassical statistical model for quantum dissipation," Physica A, vol. 236, no. 3-4, pp. 429-447, 1997.

[13] M. A. Porter and R. L. Liboff, "Vibrating quantum billiards on Riemannian manifolds," International Journal of Bifurcation and Chaos in Applied Sciences and Engineering, vol. 11, no. 9, pp. 2305-2315, 2001.

[14] T. C. Blum and H.-T. Elze, "Semiquantum chaos in the double well," Physical Review E, vol. 53, no. 4, pp. 3123-3133, 1996.

[15] L. L. Bonilla and F. Guinea, "Collapse of the wave packet and chaos in a model with classical and quantum degrees of freedom," Physical Review A, vol. 45, no. 11, pp. 7718-7728, 1992.

[16] R. I. Cukier and M. Morillo, "Comparison between quantum and approximate semiclassical dynamics of an externally driven spin-harmonic oscillator system," Physical Review A, vol. 61, no. 2, 4 pages, 2000.

[17] A. K. Pattanayak and W. C. Schieve, "Semiquantal dynamics of fluctuations: ostensible quantum chaos," Physical Review Letters, vol. 72, no. 18, pp. 2855-2858, 1994.

[18] A. M. Kowalski, M. T. Martin, J. Nuñez, A. Plastino, and A. N. Proto, "Semiquantum chaos and the uncertainty principle," Physica A, vol. 276, no. 1, pp. 95-108, 2000.

[19] A. M. Kowalski, M. T. Martin, A. Plastino, A. N. Proto, and O. A. Rosso, "Wavelet statistical complexity analysis of the classical 
limit," Physics Letters, Section A, vol. 311, no. 2-3, pp. 180-191, 2003.

[20] F. Cooper, J. F. Dawson, D. Meredith, and H. Shepard, "Semiquantum chaos," Physical Review Letters, vol. 72, no. 9, pp. 13371340, 1994.

[21] A. M. Kowalski, A. Plastino, and A. N. Proto, "Classical limits," Physics Letters, Section A, vol. 297, no. 3-4, pp. 162-172, 2002.

[22] C. M. Sarris and A. N. Proto, "Information entropy and nonlinear semiquantum dynamics," International Journal of Bifurcation and Chaos, vol. 19, no. 10, pp. 3473-3484, 2009.

[23] A. M. Kowalski, M. T. Martin, A. Plastino, and A. N. Proto, "Classical limit and chaotic regime in a semi-quantum Hamiltonian," International Journal of Bifurcation and Chaos in Applied Sciences and Engineering, vol. 13, no. 8, pp. 2315-2325, 2003.

[24] C. M. Sarris, F. Caram, and A. N. Proto, "The uncertainty principle as invariant of motion for time-dependent Hamiltonians," Physics Letters, Section A, vol. 324, no. 1, pp. 1-8, 2004.

[25] Y. Alhassid and R. D. Levine, "Entropy and chemicalchange. III. The maximal entropy (subject to constraints) procedure as a dynamical theory," The Journal of Chemical Physics, vol. 67, no. 10, 1977.

[26] Y. Alhassid and R. D. Levine, "Connection between the maximal entropy and the scattering theoretic analyses of collision processes," Phys. Rev. A, vol. 18, no. 1, pp. 89-116, 1978.

[27] D. Otero, A. Plastino, A. N. Proto, and G. Zannoli, "Ehrenfest theorem and information theory," Physical Review A, vol. 26, no. 3, pp. 1209-1217, 1982.

[28] E. Merzbaher, Quantum Mechanics, Wiley, 1963.

[29] W.-K. Tung, Group Theory in Physics, World Scientific Publishing, Singapore, 1985.

[30] W. Cheney and D. Kincaid, Métodos Numéricos y Computación, CENGAE Learning, Mexico, 2011. 


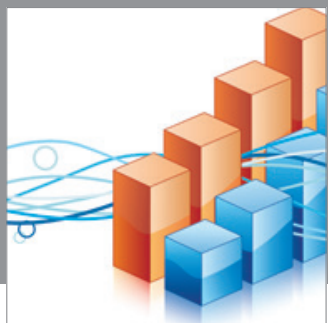

Advances in

Operations Research

mansans

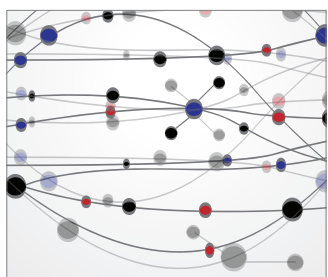

The Scientific World Journal
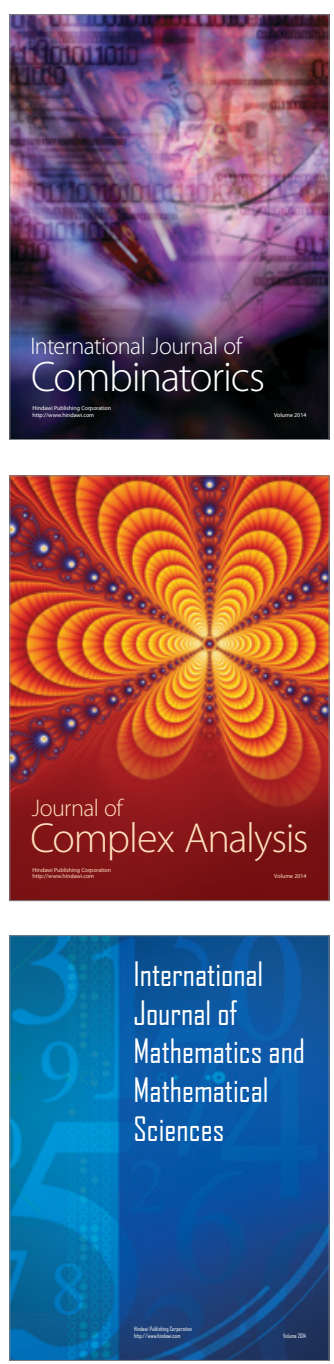
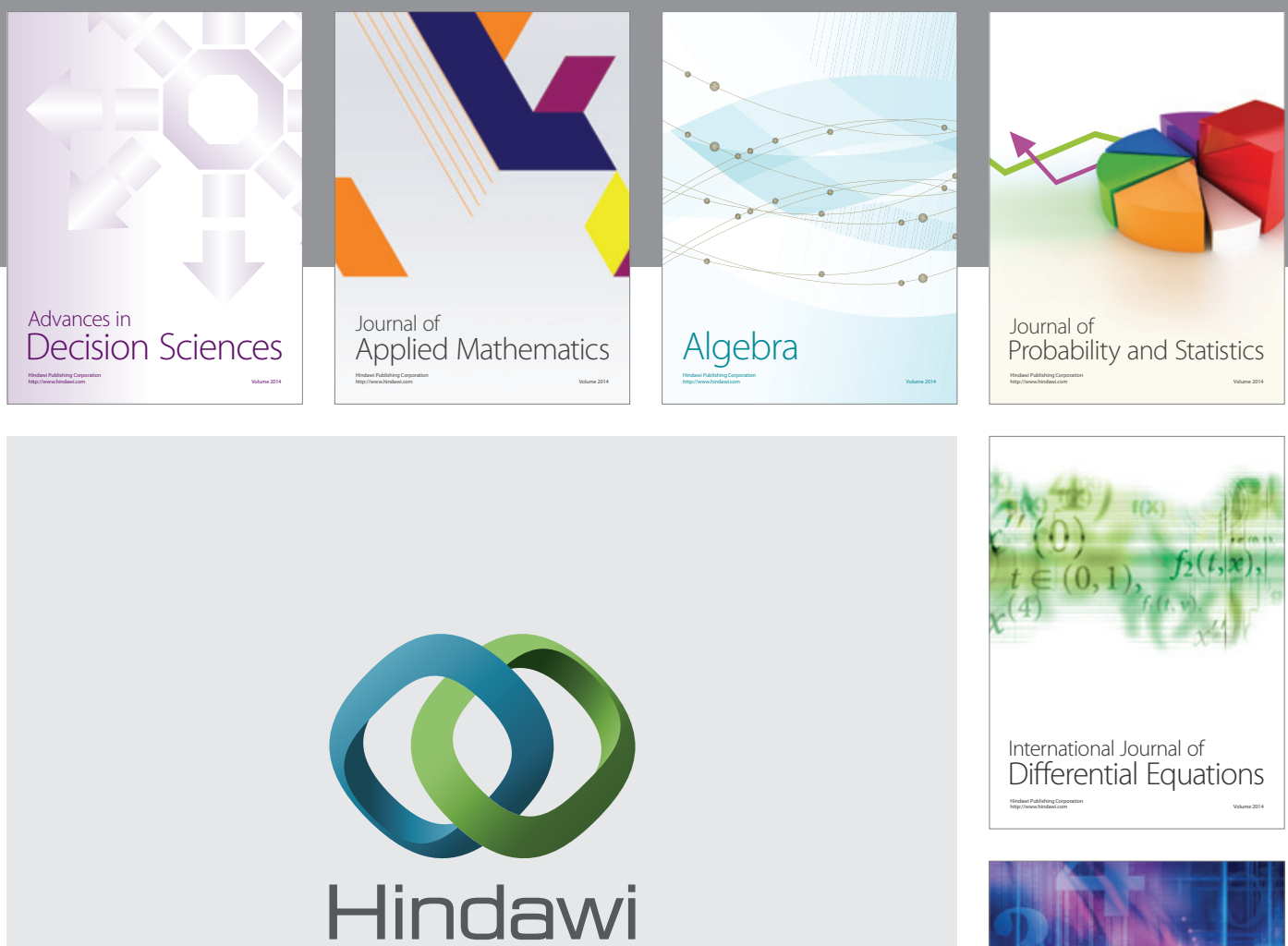

Submit your manuscripts at http://www.hindawi.com
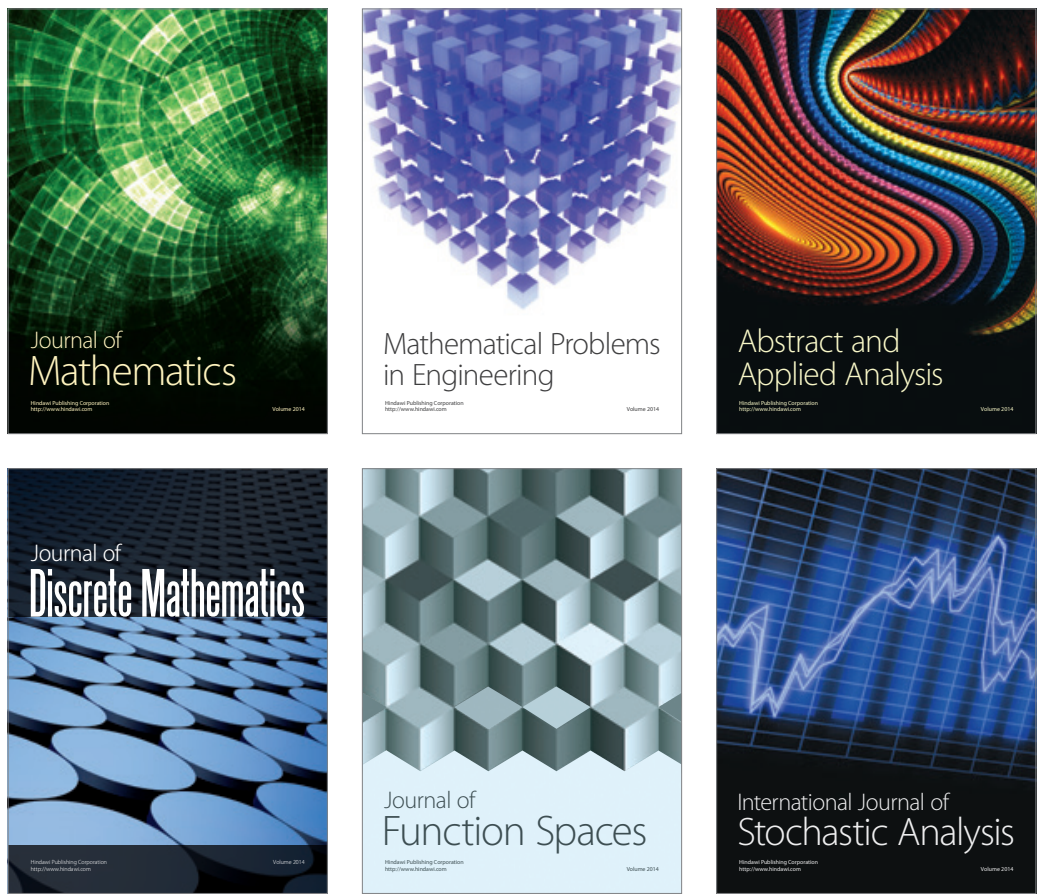

Journal of

Function Spaces

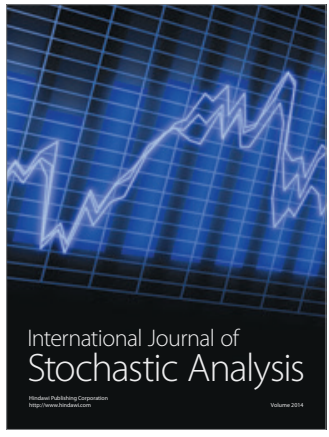

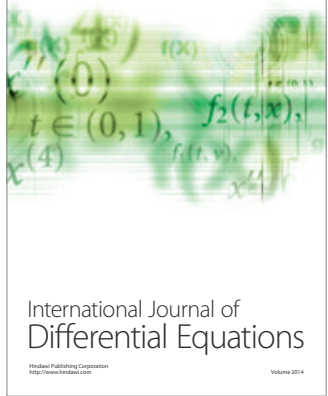
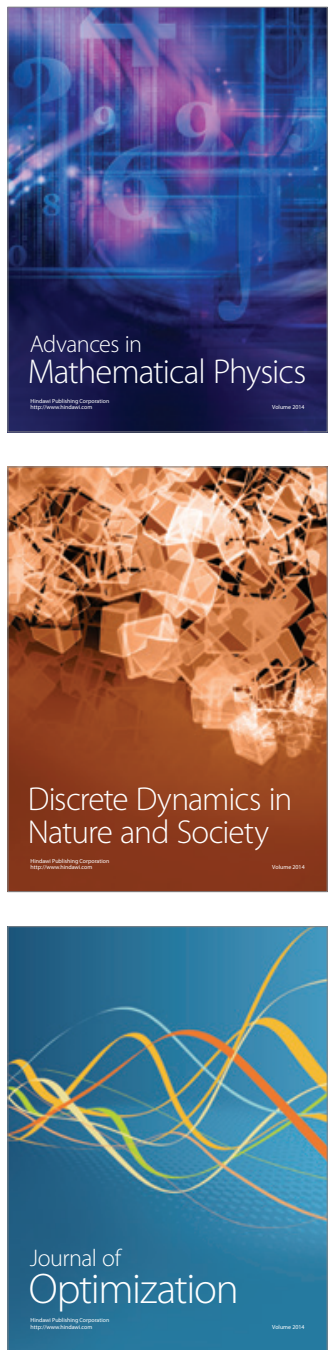